\title{
The sustained effect (I 2 months) of a single-dose vectored thermal pulsation procedure for meibomian gland dysfunction and evaporative dry eye
}

\author{
This article was published in the following Dove Press journal: \\ Clinical Ophthalmology \\ 26 July 2016 \\ Number of times this article has been viewed
}

\section{Caroline A Blackie' \\ Christy A Coleman' \\ Edward J Holland ${ }^{2}$ \\ On behalf of the LipiFlow Study Group \\ 'TearScience Inc., Morrisville, NC, ${ }^{2}$ Cincinnati Eye Institute, Edgewood, KY, USA}

Purpose: To evaluate the sustained effect (up to 1 year) of a single, 12-minute vectored thermal pulsation (VTP) treatment in improving meibomian gland function and dry eye symptoms in patients with meibomian gland dysfunction and evaporative dry eye.

Methods: The prospective, multicenter, open-label clinical trial included 200 subjects (400 eyes) who were randomized to a single VTP treatment (treatment group) or twice-daily, 3-month, conventional warm compress and eyelid hygiene therapy (control group). Control group subjects received crossover VTP treatment at 3 months (crossover group). Effectiveness measures of meibomian gland secretion (MGS) and dry eye symptoms were evaluated at baseline and 1, 3, 6,9 , and 12 months. Subjects with inadequate symptom relief could receive additional meibomian gland dysfunction therapy after 3 (treatment group) and 6 months (crossover group).

Results: At 3 months, the treatment group had greater mean improvement in MGS $(P<0.0001)$ and dry eye symptoms ( $P=0.0068)$, compared to controls. At 12 months, $86 \%$ of the treatment group had received only one VTP treatment, and sustained a mean improvement in MGS from $6.4 \pm 3.7$ (baseline) to $17.3 \pm 9.1(P<0.0001)$ and dry eye symptoms from $44.1 \pm 20.4$ to $21.6 \pm 21.3$ $(P<0.0001) ; 89 \%$ of the crossover group had received only one VTP treatment with sustained mean improvement in MGS from $6.3 \pm 3.6$ to $18.4 \pm 11.1(P<0.0001)$ and dry eye symptoms from 49.1 \pm 21.0 to $24.0 \pm 23.2(P<0.0001)$. Greater mean improvement in MGS was associated with less severe baseline MGS $(P=0.0017)$ and shorter duration of time between diagnosis and treatment $(P=0.0378)$.

Conclusion: A single VTP treatment can deliver a sustained mean improvement in meibomian gland function and mean reduction in dry eye symptoms, over 12 months. A single VTP treatment provides significantly greater mean improvement in meibomian gland function and dry eye symptoms as compared to a conventional, twice-daily, 3-month regimen. Early VTP intervention for meibomian gland dysfunction is associated with improved treatment outcomes.

Keywords: meibomian gland dysfunction, dry eye, vectored thermal pulsation, LipiFlow ${ }^{\circledR}$

\section{Introduction}

Dry eye is currently understood to be a multifactorial disease state that afflicts many millions of people worldwide. ${ }^{1}$ While our awareness of the disease has expanded exponentially since it was first documented in $1950,{ }^{2}$ as a collective, we continue to struggle with formulating a comprehensive definition for this disease. ${ }^{3}$ Currently, the definition is constrained to that of measureable dry eye sequelae with no mention of an etiology. Despite the limitations of the current definition of dry eye, there is strong
Correspondence: Caroline A Blackie TearScience Inc., 400 Commonwealth Ave, Unit \#2, Boston, MA 02215, USA Tel + I 6174236370

Fax + I 6174264924

Email cblackie@tearscience.com (c) (1) (5) 2016 Blackie et al. This work is published and licensed by Dove Medical Press Limited. The full terms of this license are available at https://www.dovepress.com/terms.php cc) ${ }_{\mathrm{BY}} \mathrm{NC}$ and incorporate the Creative Commons Attribution - Non Commercial (unported, v3.0) License (http://creativecommons.org/licenses/by-nc/3.0/). By accessing the work you hereby accept the Terms. Non-commercial uses of the work are permitted without any further permission from Dove Medical Press Limited, provided the work is properly attributed. For permission for commercial use of this work, please see paragraphs 4.2 and 5 of our Terms (https://www.dovepress.com/terms.php). 
consensus that meibomian gland dysfunction (MGD) is likely the leading cause of dry eye. ${ }^{4,5}$

This recent conclusion by the prestigious MGD Workshop report ${ }^{5}$ that MGD is likely the leading cause of all dry eye is supported by a large body of evidence-based medicine reporting that compromise to meibomian gland function negatively impacts all aspects of ocular surface health. ${ }^{4-10}$ This awareness has thus impacted dry eye management in general, not only dry eye secondary to MGD. As an example, there are a growing number of reports indicating that patients with refractory dry eye, including those with Sjogren's syndrome, show significant improvement when they receive effective therapy for MGD. ${ }^{3,11,12}$ These findings are in alignment with a statement that Wolff made in 1946 when he concluded that the meibomian glands "are essentially the glands proper to the cornea, which in the interests of vision have been moved out of the way." ${ }^{\prime 13}$ The clinical science to substantiate that the health of the ocular surface is indeed dependent upon healthy meibomian gland function has taken several decades to emerge..$^{914-16}$ The result is a growing awareness that ocular surface health cannot be sustained, optimized, or rehabilitated in the absence of a healthy meibomian gland function., ${ }^{3,17}$

It has been known for over 150 years that optimal treatment of MGD requires that some method of evacuating meibomian gland contents be employed. ${ }^{18-21}$ In combination with the core-therapeutic step of gland evacuation, or as a limited home therapy, warm compresses for MGD continue to be recommended. ${ }^{22-25}$ (Warm compresses, or other methods of front surface lid heating, do not evacuate gland contents and are, therefore, best considered adjunctive therapy. ${ }^{17}$ ) Prior to the introduction of vectored thermal pulsation (VTP) therapy, the limitation of in-office gland evacuation was the pain caused by forceful gland evacuation. ${ }^{26}$

VTP therapy was specifically designed to bypass obstacles to heat transfer and simultaneously evacuate the gland contents while heating the glands to therapeutic levels, $\geq 40^{\circ} \mathrm{C} .{ }^{6,23}$ Described fully elsewhere, the LipiFlow ${ }^{\circledR}$ System (TearScience Inc., Morrisville, $\mathrm{NC}, \mathrm{USA})$ applies heat $\left(42.5^{\circ} \mathrm{C}\right)$ to both inner eyelid surfaces (insulating the eye from the heat) while pulsating pressure is simultaneously applied to the outer eyelids using an inflatable air bladder. ${ }^{20,27}$ This temperature allows for effective heating of the meibomian gland contents, ${ }^{6}$ while operating within a safe zone so as not to cause thermal injury. ${ }^{28,29}$ As such, the LipiFlow ${ }^{\circledR}$ System is designed to evacuate the meibomian glands of the upper and lower eyelids simultaneously and with minimal discomfort, on average. ${ }^{20,27}$

The numerous peer-reviewed reports on the LipiFlow ${ }^{\circledR}$ System in both randomized controlled and uncontrolled clinical trials consistently show that the single 12-minute procedure is surprisingly effective and that the effect can be sustained. ${ }^{17,20,30-33}$ What has not been adequately investigated is the potential for a sustained effect (longer than 6 months) of a single VTP procedure. Uncontrolled reported case series evidence the effects can last longer than 12 months (up to 3 years following a single treatment ${ }^{33}$ ) but these results remain open to question due to the absence of study controls.

In addition, prior published randomized clinical trials have demonstrated the safety and effectiveness of VTP treatment for MGD as compared to conventional MGD therapy. ${ }^{17,20,30,31}$ However, only one study ${ }^{30}$ has compared a single VTP treatment to a robust, twice-daily, warm compress and lid massage home therapy, over 3 months, and the sample size in this study was small $(\mathrm{n}=31)$. Therefore, confirmation of these results with a larger study population is warranted.

Accordingly, the purpose of this study was to evaluate the long-term (up to 1 year) effectiveness in improving meibomian gland function and dry eye symptoms after a single, in-office, 12-minute VTP procedure for treatment of MGD by: 1) comparing its effectiveness to a twice-daily conventional MGD therapy over 3 months and 2) evaluating its effectiveness over 12 months.

\section{Methods}

This prospective, multicenter clinical trial was conducted in compliance with US Code of Federal Regulations (CFR): 21 CFR Parts 50, 54, and 56. The study was performed under the approval of the Oak Lawn Institutional Review Board (Oak Lawn, IL) as a nonsignificant risk postmarket study, and all tenets of the Declaration of Helsinki for the protection of human subjects in medical research were strictly observed. This study was registered at the US National Institutes of Health (ClinicalTrials.gov) \#NCT01521507. Between February 19, 2012 and October 15, 2012, a total of 200 adult subjects (400 eyes) with MGD and dry eye symptoms were randomized in the study at nine US sites. Subjects were not required to pay for their designated treatment.

\section{Study design}

The study was divided into two stages (Figure 1). Stage 1: enrollment to 3 months was an open-label, randomized controlled study design to compare the effectiveness of a single VTP treatment with the LipiFlow ${ }^{\circledR}$ System (treatment group) to a standardized twice-daily warm compress and eyelid hygiene control therapy (control group). At the end of stage 1 (3 months) was a crossover VTP treatment of the control subjects (crossover group). Stage 2: between 3 and 


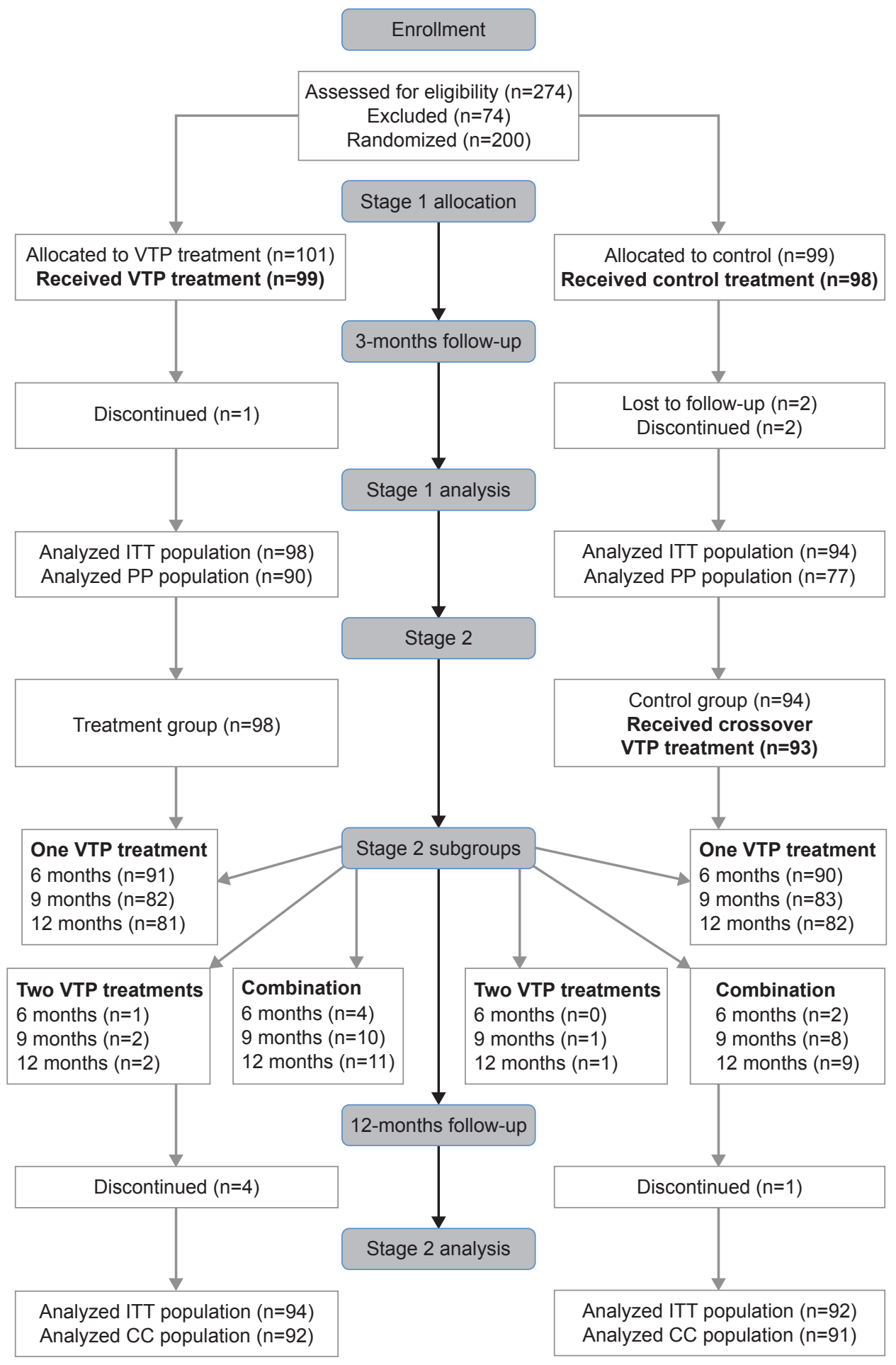

Figure I Abbreviated CONSORT flow diagram illustrating the subject disposition from enrollment through the completion of stage 2 of the study. Abbreviations: CC, completed cases; ITT, intention-to-treat; PP, per protocol; VTP, vectored thermal pulsation.

12 months, an observational study was performed to evaluate the potential sustained effect of a single VTP treatment alone or in combination with other MGD and dry eye treatments deemed necessary during the study period. Subjects were entered into the following subgroups based on the subject's self-assessment of the adequacy of symptom relief and the physician's assessment that additional treatment was or was not necessary. 1) One VTP treatment: subjects received only one VTP treatment; 2) two VTP treatments: subjects received two VTP treatments; 3) combination treatment: subjects 
received one or two VTP treatments followed by other prescribed MGD or dry eye treatment. All subjects provided written informed consent prior to study participation.

\section{Inclusion criteria}

The inclusion criteria were as follows: Age at least 18 years; willing to comply with the study procedures and follow-up; evidence of meibomian gland obstruction (based on a meibomian gland secretion (MGS) score of $\leq 12$ for 15 glands of the lower lid) in both eyes; reported dry eye symptoms within the past 3 months and a score $\geq 6$ in both eyes using the Standard Patient Evaluation of Eye Dryness questionnaire; ${ }^{34}$ Ocular Surface Disease Index (OSDI) questionnaire score $\geq 13$ in both eyes, ${ }^{35}$ and mean lipid layer thickness of $\leq 80 \mathrm{~nm}$ in both eyes. ${ }^{34}$

\section{Exclusion criteria}

Exclusion criteria were conditions that could potentially increase the risk of a procedure-related injury, limit effectiveness of the study treatment, or interfere with the assessment of effectiveness. These included active ocular infection or inflammation (including allergic, vernal, or giant papillary conjunctivitis and severe eyelid inflammation); ocular surface abnormality that may compromise corneal integrity; eyelid abnormalities that affect lid function; recent (within the past 3 months) ocular surgery, trauma, herpes, or recurrent inflammation; systemic disease condition that causes dry eye; punctal plugs or punctal occlusion within the past 3 months; unwilling to abstain from the use of systemic medications known to cause dryness for the study duration; unwilling to abstain from the use of systemic or topical treatments for MGD or dry eye for the study duration (except for over-the-counter [OTC] artificial tears, ocular lubricants, or dietary supplements); pregnant, nursing, or females of childbearing potential and not utilizing adequate birth control measures; and participation in another ophthalmic clinical trial involving a therapeutic drug or device within the past 30 days. Prior to the baseline visit, subjects were required to discontinue use of systemic antihistamines or isotretinoin for at least 1 month, cyclosporine ophthalmic emulsion (Restasis $^{\circledR}$; Allergan, Inc., Irvine, CA, USA) for at least 2 months, all other prescription medications used for dry eye or MGD (eg, antibiotics, corticosteroids, and non-steroidal anti-inflammatory drugs) for at least 2 weeks, and all other MGD treatments (eg, at-home warm compress therapy, eyelid hygiene, eyelid massage, and manual lid expression) for at least 2 weeks.

\section{Randomization and subject disposition}

A total of 101 subjects (202 eyes) were randomized to the treatment group (VTP treatment) and 99 subjects (198 eyes) were randomized to the control group (warm compress and eyelid hygiene). Subjects in the treatment group received a single 12-minute VTP treatment and those in the control group were instructed to perform twice-daily, 10-minute warm compress, and eyelid hygiene treatment of both eyes for 3 months, which was standardized using the OTC EyeGiene $^{\circledR}$ Insta-Warmth ${ }^{\text {TM }}$ System (Eyedetec Medical, Inc. Danville, CA, USA) and OCuSOFT ${ }^{\circledR}$ Lid Scrub $^{\text {TM }}$ Original (OCuSoft, Rosenberg, TX, USA). Control subjects documented daily therapy use on a diary. In stage 1 , treatment and control group subjects were followed at 1 and 3 months after the treatment visit. After the 3-month exam, control subjects were instructed to stop the control therapy and received crossover VTP treatment. In stage 2, treatment group subjects were followed at 6, 9, and 12 months. Control subjects who became crossover group subjects were followed at 4, 6, 9, and 12 months. The complete subject disposition is given in the form of an abbreviated Consolidated Standards of Reporting Trials flow diagram in Figure 1.

During the study, the use of any prescribed medication or treatment as deemed necessary by the physician to alleviate an adverse event was allowed. In addition, subjects were permitted the use of OTC supplements during the study. No prescription MGD or dry eye treatment was permitted for treatment group subjects for the first 3 months and for control/crossover group subjects through the first 6 months. In stage 2, subjects were assessed at each visit for adequacy of symptom relief to determine which subgroup the subjects entered for the subsequent visit. Subjects who reported adequate symptom relief remained in the one VTP subgroup, and no additional treatment was prescribed until the next visit, when the adequacy of symptom relief was assessed again. Subjects who reported inadequate symptom relief were provided either a second VTP treatment (two VTP subgroups) or other MGD and dry eye treatment (combination subgroup). If the subject self-initiated other MGD or dry eye treatment during the study (except for OTC supplements), the subject was entered into the combination subgroup and was no longer eligible for a second VTP treatment. For the combination subgroup, the type of other MGD or dry eye treatments prescribed by the physician included warm compress, lid hygiene, lid massage, systemic antibiotic, topical antibiotic, and topical steroid. 


\section{The LipiFlow ${ }^{\circledR}$ System}

The LipiFlow ${ }^{\circledR}$ Vectored Thermal Pulsation System is a prescription device intended for the application of localized heat and pressure therapy in adult patients with chronic cystic conditions of the eyelids, including MGD, also known as evaporative dry eye or lipid deficiency dry eye. The LipiFlow ${ }^{\circledR}$ System is used by a physician in an in-office procedure to provide controlled heat to the inner eyelid surface and intermittent pressure to the outer eyelid to facilitate release of lipid from the glands. The device has been described in detail elsewhere. ${ }^{20}$

\section{Study parameters}

Study effectiveness endpoint parameters were Meibomian Gland Assessment and the OSDI Questionnaire. Meibomian gland assessment was performed using a handheld instrument, Meibomian Gland Evaluator, to apply standardized pressure to the eyelid margin, which simulates a blink in yielding gland secretions. A total of 15 glands were evaluated along the lower eyelid margin, consisting of five glands located in each of the temporal, central, and nasal regions. For each of the 15 glands, expressed secretion characteristics were graded as 3 (clear liquid secretion), 2 (cloudy liquid secretion), 1 (inspissated/toothpaste consistency), and 0 (no secretion). For data analysis, the MGS score for each eye was calculated based on the sum of the secretion grades for all 15 glands evaluated with a range of 0 to 45 .

The OSDI questionnaire assessed the subjects' frequency of dry eye symptoms and problems with their eyes in situations and conditions of daily living. The dry eye questionnaire was explained to the subjects prior to completion to ensure understanding of the instructions without coaching or influencing the subjects' responses. The subjects completed the questionnaire directly and signed and dated the form. The OSDI score was calculated as the sum of frequency scores for all symptoms multiplied by 25 and divided by the number of questions answered with a range from 0 to $100 .{ }^{33}$

\section{Study endpoints}

Stage 1 primary and secondary effectiveness endpoints were to compare the mean change from baseline to 3 months in MGS and OSDI scores, respectively, between the treatment and control groups. Stage 2 primary and secondary effectiveness endpoints were to evaluate the sustained treatment effect at 6 and 12 months based on the mean MGS score and mean OSDI score, respectively, with comparison between the stage 2 subgroups. There was no safety endpoint in this study because the LipiFlow ${ }^{\circledR}$ System is a nonsignificant risk device and safety has been demonstrated through a previous study. ${ }^{20}$ However, adverse event data were collected at all study visits.

\section{Study populations}

Three populations were used for analysis. 1) The intentionto-treat (ITT) population included all subjects who were randomized in the study and was used for analysis of the stage 1 primary and secondary endpoints and adverse events. 2) The per protocol (PP) population, which included all subjects who completed stage 1 with no significant protocol deviations that could affect the effectiveness endpoints, was also used for stage 1 endpoint analyses. Control subjects who did not meet the minimum acceptable compliance for use of the control therapy as defined in the protocol were excluded from the PP population. Of the control subjects who completed the 3 -month visit, $84.0 \%$ met the minimum acceptable compliance. 3) The completed cases (CC) population included all subjects who completed 12 months and was analyzed for the stage 2 primary and secondary effectiveness endpoints.

\section{Statistical analysis}

Statistical analysis was performed using SAS (SAS Institute Inc., Cary, NC, USA) software version 9.2. A comparability analysis by treatment group was performed for demographics (age, sex, race, and ethnicity) and baseline characteristics (MGS score, OSDI score, duration of dry eye symptoms and duration of dry eye, or MGD diagnosis). Wilcoxon rank sum test was used for comparison of continuous variables. For categorical variables, Fisher's exact test or Fisher-FreemanHalton test was used. A $P<0.1$ was used to determine imbalance between groups. Any significant variables were included in supportive multivariate models of study endpoints to control for the imbalance.

To assess poolability of the multicenter data, a multivariate mixed models analysis of variance of the stage 1 primary and secondary effectiveness endpoints was employed to test whether a site by treatment group interaction existed. A two-sided test with a $P$-value $<0.10$ was used to determine a significant site by treatment interaction. If the effect size was homogeneous by site, the stage 1 endpoint was analyzed by a two-sample $t$-test. If the effect size was heterogeneous by site, the overall effect size across sites for the stage 1 endpoint was obtained by taking the weighted average of the difference in scores between groups at each site, using the inverse of the variance of the difference in scores as the 
weight, and dividing the sum of these weighted differences by the sum of the weights. The test of significance was a z-statistic. To avoid multiplicity issues, the stage 1 primary and secondary endpoints were tested in order under a closed form testing method. The secondary hypothesis was tested only if the primary hypothesis was statistically significant. If both hypotheses were statistically significant, the overall study alpha is 0.025 .

Supportive multivariate generalized linear mixed models for the stage 1 endpoints were analyzed with covariates, including treatment group, demographics, baseline characteristics, and any variables found to be out of balance from the comparability analyses. The data were modeled based on subject as the independent unit of measure to account for any clustering and correlation between right and left eyes of each subject.

Stage 2 primary and secondary effectiveness endpoints were analyzed with a multivariable mixed model with stage 2 subgroup as a fixed effect. Covariates for the model included treatment group, demographics, and baseline characteristics. Stage 2 endpoints were analyzed at 12 months with comparison of the one VTP subgroup to the combination subgroup only because there were too few subjects in the two VTP subgroup ( $\mathrm{n}=3$ subjects) at 12 months for meaningful analysis. Stage 2 endpoints were not analyzed at 6 months because there were too few subjects in the two VTP ( $\mathrm{n}=1$ subject) and combination ( $n=6$ subjects) subgroups at 6 months for a meaningful comparative analysis.

Exploratory analyses included analysis of the sustained effect by assessing the mean change in MGS and OSDI scores from baseline to 12 months using a paired $t$-test and the CC population of the one VTP subgroup in the treatment and crossover groups. In addition, to determine patient characteristics significantly associated with greater MGS improvement after VTP treatment, the change in MGS score from baseline to 3 months was analyzed for the treatment group using a multivariate mixed model and the ITT population. Model covariates included demographics and baseline characteristics.

\section{Results}

The mean age of the subjects was $56.2 \pm 15.3$ years (range: 22 to 85 years) with $71 \%$ females. Most subjects were of non-Hispanic or non-Latino ethnicity (93.0\%) and White or Caucasian race $(97.0 \%)$. There were no statistically significant differences $(P>0.10)$ in demographics, baseline total MGS score or duration of MGD, or dry eye diagnosis between the treatment and control groups (Table 1). The retention of subjects over the study duration was excellent. Of the 200 randomized subjects, 192 (96.0\% accountability) completed stage 1 (3 months) and 186 (93.0\% accountability) completed stage 2 (12 months).

\section{Stage I primary and secondary effectiveness endpoints}

Table 2 displays the mean change in MGS score and OSDI score for the treatment and control groups from baseline to 3 months for the ITT and PP populations. The treatment group had a statistically significant greater mean improvement in MGS score $(P<0.0001)$ between baseline and 3 months as compared to the control group after accounting for heterogeneity across sites. Furthermore, the treatment group had a statistically significant greater mean reduction in OSDI score ( $P=0.0068$ for ITT population; $P=0.0018$ for PP population) between baseline and 3 months as compared to the control group. In the supportive multivariate models of the stage 1 endpoints controlling for demographic and baseline covariates (including imbalanced variables from the comparability analysis), the treatment group showed a statistically significant greater mean improvement in MGS score

Table I Baseline characteristics for treatment and control groups

\begin{tabular}{llll}
\hline Group/baseline characteristic & Treatment & Control & Treatment vs control $^{\mathrm{a}}$ \\
\hline Duration of dry eye symptoms (years) & $\mathrm{N}=10$ I subjects & $\mathrm{N}=98$ subjects $^{\mathrm{b}}$ & $P=0.0889$ \\
Duration MGD/dry eye diagnosis (years) & $6.9 \pm 6.8$ & $8.7 \pm 7.7$ & $\mathrm{~N}=0.8589$ \\
Baseline MGS score & $\mathrm{N}=83$ subjects & \\
& $5.3 \pm 6.2$ & $\mathrm{~N}=85$ subjects & \\
Baseline OSDI score & $\mathrm{N}=202$ eyes & $5.5 \pm 5.9$ & $\mathrm{~N}=0.727 \mathrm{I}$ \\
& $6.2 \pm 3.7$ & $6.3 \pm 3.7$ & $\mathrm{P}=0.0734$ \\
\hline
\end{tabular}

Notes: a $P$-value based on Wilcoxon rank sum test; $P<0.10$ was used to determine imbalance between groups. 'Duration of dry eye symptoms was reported as "unknown" for one control subject. Duration of MGD or dry eye diagnosis was reported as "unknown" for 18 treatment subjects and I4 control subjects. Data are presented as mean \pm standard deviation.

Abbreviations: MGD, meibomian gland dysfunction; MGS, meibomian gland secretion; OSDI, Ocular Surface Disease Index. 
Table 2 Mean \pm standard deviation change in MGS score and OSDI score from baseline to 3 months between treatment and control groups

\begin{tabular}{llllllll}
\hline Parameter & Population & N & Treatment & N & Control & Effect size & P-value \\
\hline MGS & Intention-to-treat & 196 & $11.6 \pm 9.9$ & 188 & $4.5 \pm 7.8$ & 5.7 & $<.6$ \\
& Per protocol & 177 & $11.2 \pm 9.3$ & 153 & $4.5 \pm 8.2$ & $<0.000 I^{\mathrm{a}}$ \\
\multirow{2}{*}{ OSDI } & Intention-to-treat & 196 & $-23.4 \pm 20.6$ & 188 & $-17.8 \pm 19.9$ & -5.6 & $<0.000 I^{\mathrm{a}}$ \\
& Per protocol & 177 & $-24.0 \pm 20.9$ & 153 & $-17.1 \pm 18.6$ & -6.9 & $0.0068^{\mathrm{b}}$ \\
& &
\end{tabular}

Notes: ${ }^{\text {a }}$-value based on z-statistic of the sum of weighted average of difference in scores between groups at each site divided by the sum of the weights. ${ }^{b} P$-value based on two sample $t$-test with a one-sided $\alpha=0.025$.

Abbreviations: MGS, meibomian gland secretion; N, number of eyes; OSDI, Ocular Surface Disease Index.

$(P=0.0020)$ and OSDI score $(P=0.0419)$ between baseline and 3 months as compared to the control group.

\section{Stage 2 primary and secondary effectiveness endpoints}

Stage 2 subgroup classification and posttreatment care regimen depended upon the subject's report of adequacy of symptom relief at 3,6, and 9 months for treatment group subjects and at 6 and 9 months for crossover group subjects. Most treatment group subjects remained in the one VTP subgroup at 6 months (94.8\%), 9 months (87.2\%), and 12 months $(86.2 \%)$. Similarly, most crossover group subjects remained in the one VTP subgroup at 6 months (97.8\%), 9 months (90.2\%), and 12 months (89.1\%). Subjects with inadequate symptom relief moved from the one VTP subgroup to the two VTP and/or combination subgroups over time. At 12 months, $11.7 \%$ and $9.8 \%$ of subjects in the treatment and crossover groups, respectively, were in the combination subgroup, and $2.1 \%$ and $1.1 \%$ of subjects in the treatment and crossover groups, respectively, were in the two VTP subgroup.

Table 3 displays the mean MGS score and mean OSDI score at 12 months with comparison between the one VTP and combination subgroups for the $\mathrm{CC}$ population. In the multivariate model controlling for other covariates, there was no statistically significant difference in the 12-month mean MGS score between one VTP and combination subgroups ( $P=0.9098)$. Conversely, the one VTP subgroup had a statistically significantly lower (less dry eye symptoms) mean
OSDI score at 12 months as compared to the combination subgroup ( $P=0.0237$ ), after controlling for the baseline OSDI score and other covariates in the multivariate model. No significant difference was found between treatment and crossover groups at 12 months in MGS score $(P=0.7062)$ or OSDI score ( $P=0.8773)$, supporting comparable outcomes in the treatment and crossover groups.

\section{Sustained effect over 12 months}

For the 12-month cohort (CC population) of the one VTP subgroup in both the treatment and crossover groups, the mean MGS score over time is shown in Figure 2 and the mean OSDI score over time is shown in Figure 3. The treatment group received only one VTP treatment and no other prescribed MGD or dry eye therapy. The results reflect a significant and sustained mean improvement in MGS score from baseline to 12 months $(6.4 \pm 3.7$ to $17.3 \pm 9.1 ; P<0.0001)$ for the $86.2 \%$ of treatment group subjects who received only one VTP treatment through 12 months. Furthermore, a significant and sustained mean reduction in OSDI score from baseline to 12 months ( $44.1 \pm 20.4$ to $21.6 \pm 21.3 ; P<0.0001$ ) was observed in treatment group subjects who received only one VTP treatment from baseline to 12 months.

The crossover group received 3 months of twice-daily warm compress and eyelid hygiene control therapy, followed by only one VTP treatment and no other prescribed MGD or dry eye therapy. While the mean MGS improved from baseline after 3 months of control therapy $(6.3 \pm 3.6$ to 11.0 \pm 8.0 ), the mean MGS improved further 1 month after

Table 3 Mean \pm standard deviation MGS score and OSDI score at 12 months for one LipiFlow ${ }^{\circledR}$ and combination subgroups

\begin{tabular}{lllllll}
\hline Parameter & Group & $\mathbf{N}$ & One VTP subgroup & $\mathbf{N}$ & Combination subgroup & P-value* \\
\hline MGS & Treatment & 162 & $17.3 \pm 9.1$ & 22 & $17.9 \pm 10.8$ & 0.9098 \\
& Crossover & 164 & $18.4 \pm 11.1$ & 18 & $17.4 \pm 8.5$ & \\
OSDI & Treatment & 162 & $21.6 \pm 21.3$ & 22 & $35.8 \pm 25.5$ & 0.0237 \\
& Crossover & 164 & $24.0 \pm 23.2$ & 18 & $42.2 \pm 33.6$ & \\
\hline
\end{tabular}

Note: $*$-value based on multivariate mixed model.

Abbreviations: MGS, meibomian gland secretion; N, number of eyes; OSDI, Ocular Surface Disease Index; VTP, vectored thermal pulsation. 


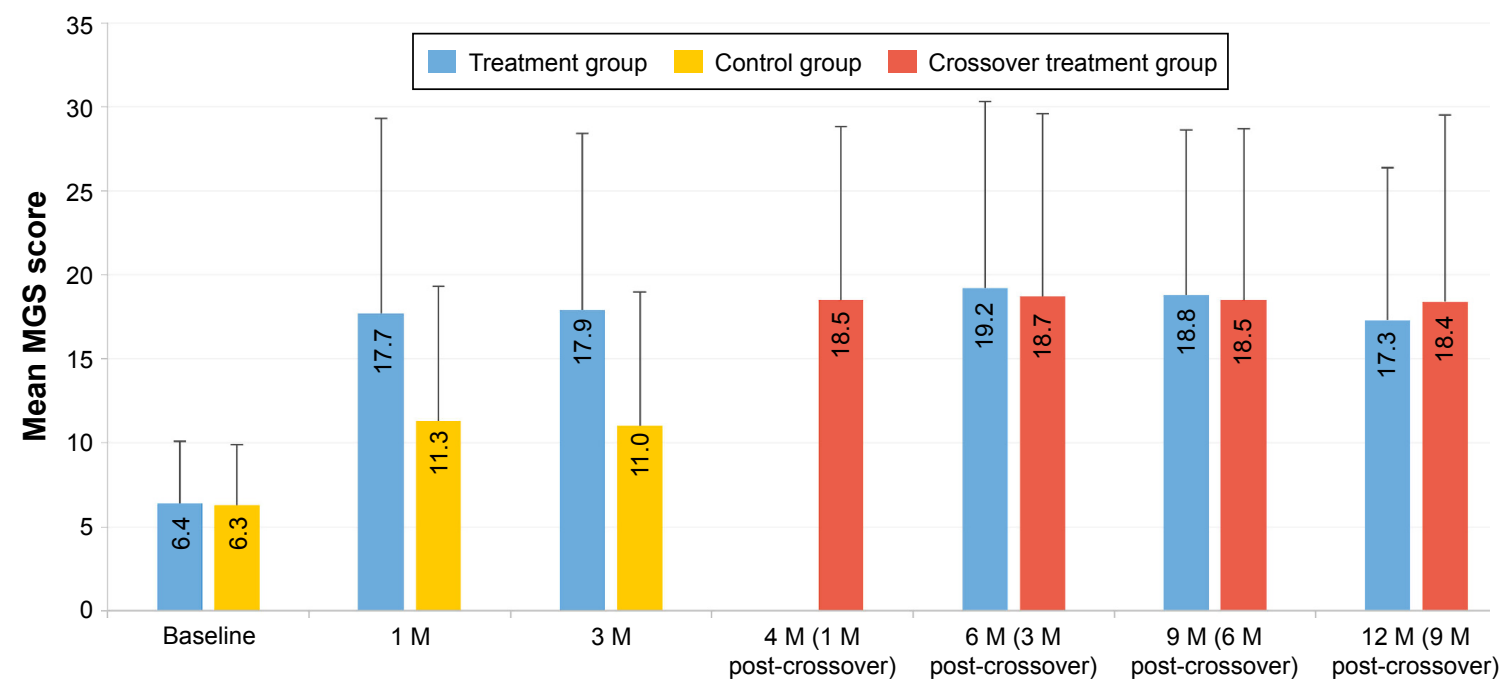

Figure 2 Mean MGS score over time for the 12-month cohort of eyes that received a single vectored thermal pulsation treatment.

Note: Error bars represent standard deviation.

Abbreviations: M, months; MGS, meibomian gland secretion.

crossover treatment at 4 months $(18.5 \pm 10.3)$, similar to the 1 month mean for the treatment group. For the $89.1 \%$ of crossover subjects who received only one $\mathrm{VTP}^{\circledR}$ treatment through 12 months, there was significant and consistent mean improvement from baseline to 12 months in MGS score $(6.3 \pm 3.6$ to $18.4 \pm 11.1 ; P<0.0001)$ and OSDI score (44.1 \pm 20.4 to $21.6 \pm 21.3 ; P<0.0001)$.

\section{Patient characteristics associated with MGS improvement}

Baseline patient characteristics that significantly associated with greater improvement in MGS score from baseline to 3 months after VTP treatment based on simple univariate models included: higher (less severe) baseline MGS score $(P=0.0007)$, shorter duration of dry eye or MGD diagnosis $(P=0.0059)$, previous history of chalazia $(P=0.0271)$, and shorter duration of dry eye symptoms $(P=0.0273)$. In the multivariate mixed model, only baseline MGS score $(P=0.0017)$ and duration of dry eye or MGD diagnosis $(P=0.0378)$ remained significantly associated with the mean change in MGS score from baseline to 3 months. Specifically, subjects with a less severe baseline MGD and/or shorter duration of time from diagnosis to treatment had a greater mean improvement in MGS score between baseline and 3 months.

Although duration of dry eye symptoms was not significant in the multivariate model, the data show the same trend

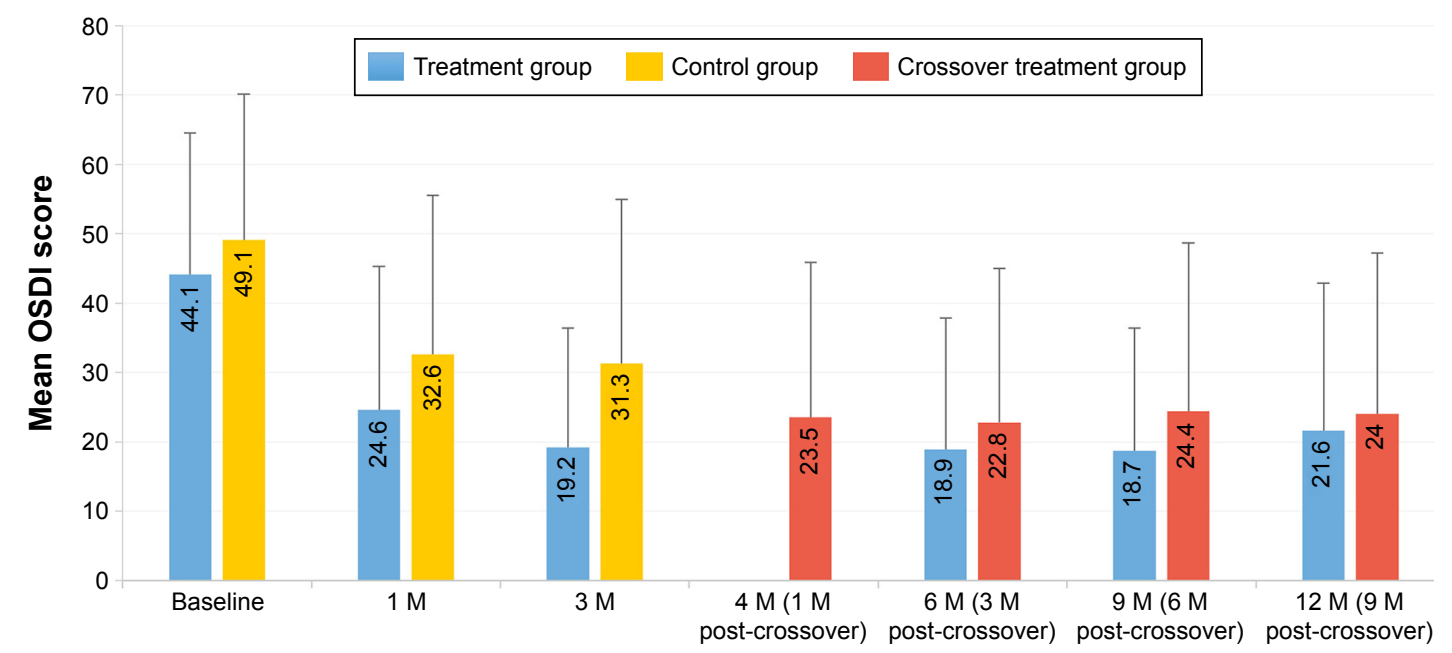

Figure 3 Mean OSDI score over time for 12-month cohort of eyes that received a single vectored thermal pulsation treatment.

Note: Error bars represent standard deviation.

Abbreviations: M, months; OSDI, Ocular Surface Disease Index. 


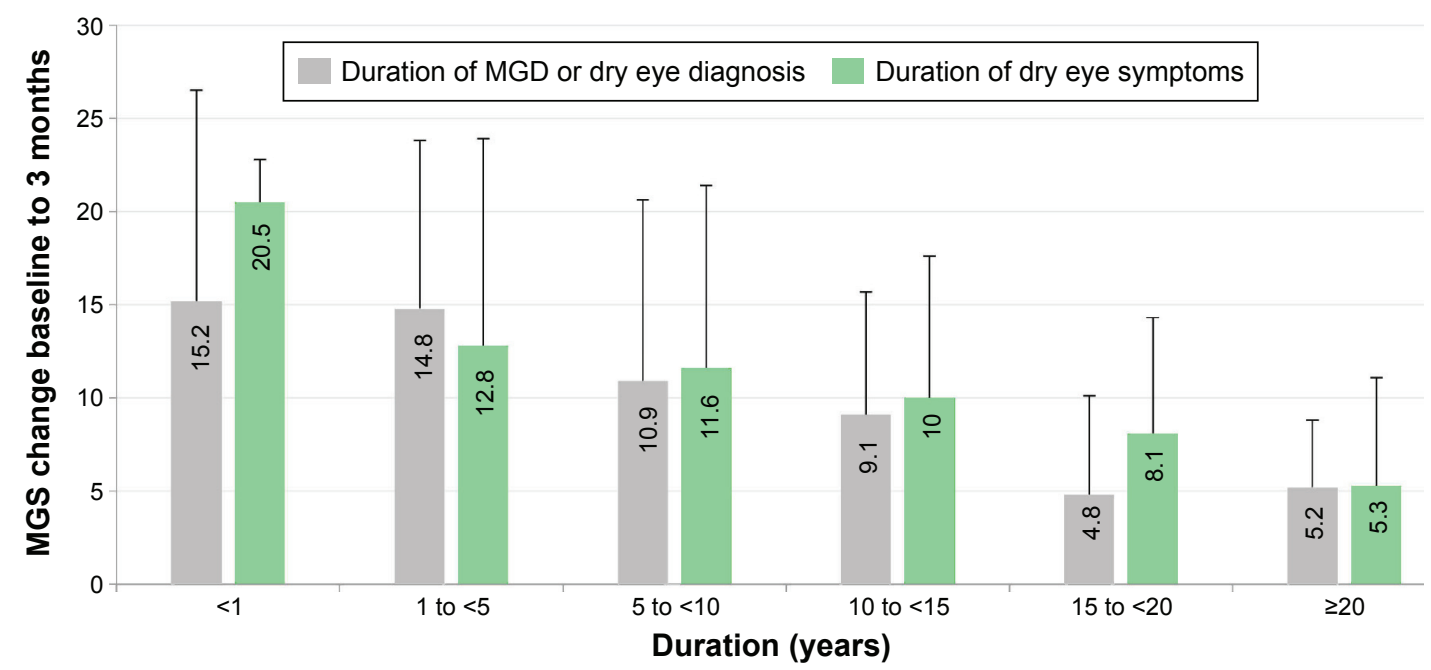

Figure 4 Mean change in MGS score from baseline to 3 months for treatment group stratified by duration of dry eye or MGD diagnosis and duration of dry eye symptoms. Note: Error bars represent standard deviation.

Abbreviations: M, months; MGD, meibomian gland dysfunction; MGS, meibomian gland secretion.

as the analogous duration of dry eye or MGD diagnosis. To illustrate the trend, Figure 4 displays the mean change in MGS score from baseline to 3 months for the treatment group stratified by duration of both diagnosis and symptom onset.

\section{Adverse events}

There were no serious device-related adverse events and no unanticipated adverse device effects reported in the study. All device-related adverse events were anticipated, transient, nonserious ocular events that resolved without sequelae. There were 19 device-related events, including ten related to VTP $^{\circledR}$ treatment, eight related to control therapy, and one related to a study procedure (combination treatment use) reported in 16 subjects (26 eyes). VTP ${ }^{\circledR}$-related events were reported for $5.1 \%$ of subjects ( $3.9 \%$ of eyes) who attempted $\mathrm{VTP}^{\circledR}$ or crossover VTP ${ }^{\circledR}$ treatment. Control-related events were reported for $7.1 \%$ of subjects ( $6.6 \%$ of eyes) who used control therapy. The most common device-related event by type was eye/eyelid discomfort/pain (1.5\%) for all $\mathrm{VTP}^{\circledR}$ treated eyes and eyelid skin dermatitis $(1.5 \%)$ for control group eyes.

\section{Discussion}

The goal of treatment for MGD is to improve meibomian gland function. Intrinsic to the definition of MGD is gland obstruction. ${ }^{4,5}$ Thus, treatment should primarily involve the evacuation of stagnated gland contents. ${ }^{17}$ Supplementary therapy, which includes front surface lid heating in various forms, lid margin scrubs, lid margin debridement-scaling, and additional disease comanagement, can then help maintain the improvement of gland function achieved by evacuating the gland contents. ${ }^{4,5,11,17,36}$ The conventional method of evacuating stagnated contents is to manually express the glands. Although the procedure is effective, it is painful and needs to be repeated several times a year. ${ }^{26}$

LipiFlow $^{\circledR}$ System is an innovative, automated VTP system, which overcomes the limitations of conventional methods and offers an effective treatment for meibomian gland obstruction. The device safely delivers a therapeutic level of heat to the palpebral surfaces of the upper and lower eyelids directly over the meibomian glands while graded pulsatile pressure simultaneously evacuates the meibomian glands during heating. ${ }^{17}$ The single, 12-minute in-office treatment has been found to be highly effective in the treatment of MGD and associated evaporative dry eye by increasing meibomian gland function, thereby improving tear break-up time, tear film stability and reducing dry eye symptoms. ${ }^{17}$

The active control in this study was standardized, twicedaily warm compress and eyelid hygiene based on the recommended standard of care for MGD and evaporative dry eye. ${ }^{36}$ The study results (Table 2 ) indicate that after 3 months, a single 12-minute VTP treatment was significantly more effective in improving meibomian gland function and reducing dry eye symptoms than twice-daily warm compress application combined with eyelid hygiene. This significant difference between treatment and control groups in these outcomes was maintained after controlling for patient demographic and baseline characteristics in the multivariate 
analysis. These results in a larger population concur with a prior report showing the efficacy of a single VTP treatment compared to a similar conventional control after 3 months of daily at-home therapy. ${ }^{30}$ While it was not possible to mask the patients to the therapy, a significant limitation of this study is that the investigators were not masked. In a prior study where the investigators were masked, the results of the treatment were clinically and statistically significant. ${ }^{30}$

The sustained effect of the single VTP treatment, with respect to meibomian gland function and dry eye symptoms, was evaluated over a 12-month period. With excellent study retention ( $93 \%$ ), a high percentage, $86.2 \%$, of treatment group subjects and $89.1 \%$ of crossover subjects remained in the one VTP subgroup until 12 months. Both treatment and crossover group subjects demonstrated consistent mean improvement in MGS score, as well as a sustained mean reduction in OSDI score over the 12-month study period (Figures 2 and 3 ). These patients did not receive any additional prescribed therapy for MGD or dry eye to further ameliorate their symptoms after the initial single VTP ${ }^{\circledR}$ treatment. This clinical trial lasted twice as long as a prior randomized controlled trial, which followed the patients who had received a single VTP treatment out to 6 months and also showed a sustained effect of the single treatment. ${ }^{31}$

In a prior report, structural gland loss in excess of $67 \%$ resulted in relatively reduced symptomatic relief and improvement in gland function compared to that of patients who had retained $33 \%$ or more of their gland structure. ${ }^{30}$ In the current study, gland imaging was not utilized to evaluate the structural integrity of the glands prior to treatment. While the correlation between gland structure and gland function is weak, gland imaging should be an integral part of any MGD workup to assist in the setting of expectations for treatment based on the findings of the prior report. ${ }^{30,31}$

MGD is understood to be a chronic progressive disease. ${ }^{17,37}$ Later stage disease increases the likelihood of irreversible anatomical changes, for example, rounding of posterior lid margin, conjunctivalization of Marx's line, meibomian gland truncation, or atrophy. ${ }^{38}$ A reasonable prediction would be that early intervention for MGD would lead to improved treatment outcomes and this view has been previously presented. ${ }^{3,17}$ As predicted, the effect of disease chronicity is demonstrated by the significant association between greater mean improvement in MGS score between baseline and 3 months and the patient characteristics of shorter duration of MGD or dry eye diagnosis and less severe baseline MGD. These results support the view that early intervention improves treatment outcomes for MGD, as measured by improvement in gland function. In addition, the baseline mean duration of dry eye symptoms was 1.6 years longer than the mean duration of diagnosis in the treatment group and 3.2 years longer in the control group (Table 1), reflecting that many patients are not diagnosed with MGD until after they are symptomatic for some time. Early intervention requires early detection, and patients should be routinely assessed for MGD even in the absence of dry eye symptoms.

In this study, a small percentage of patients received additional prescribed therapy (combination or two VTP treatments) for further symptom relief through 12 months. In the comparison of outcomes for subjects who received only one VTP treatment versus those who received combination treatment, there was no significant difference in the mean MGS score but the one VTP subjects had a statistically significantly lower mean OSDI score (less symptoms) at 12 months. Not surprisingly, the combination subjects, who were selected into this subgroup based on inadequate symptom relief, had more severe baseline dry eye symptoms on average than the one VTP subjects. In addition, the combination subjects were often not compliant with their prescribed therapy, thus limiting the conclusions that we can draw regarding these subjects. However, as other MGD or dry therapy did not further improve the mean MGS score in the combination subgroup, it is likely that factors other than MGD specifically were contributing to the greater dry eye symptoms at 12 months. This is not atypical of patients with chronic dry eye where multiple therapies may be indicated. ${ }^{22}$

The safety of the LipiFlow ${ }^{\circledR}$ System has been previously evaluated and reported. ${ }^{20}$ The type and rate of nonserious and transient adverse events related to the LipiFlow ${ }^{\circledR}$ System in this trial were consistent with prior studies.

\section{Conclusion}

A single VTP treatment can deliver a sustained effect over 1 year, as shown by mean improvement in meibomian gland function and mean reduction in dry eye symptoms from baseline. These results also show that most patients, $86.2 \%$, did not receive additional prescribed therapy for symptom relief after a single VTP treatment during the 12-month study period. Furthermore, a single VTP treatment provides significantly greater mean improvement in meibomian gland function and dry eye symptoms as compared to a robust, conventional regimen used twice-daily for 3 months. These results also suggest that early intervention for MGD improves treatment outcomes. This study strongly supports a previously published perspective that no other single dose therapy offers a comparable efficacy profile for reducing dry eye symptoms and improving meibomian gland function as well as VTP for MGD. ${ }^{17}$ 


\section{Acknowledgments}

This study was funded by TearScience, Morrisville, NC, USA. This study was conducted with the help of the LipiFlow $^{\circledR}$ Study Group: Raman Bedi, MD (Iris Advanced Eye Centre, CMC Hospital, Chandigarh, India); Chad L Betts, MD, RPh (MacDonald Eye, Fayetteville, AR); Joseph P Gira, MD (Ophthalmology consultants, Ltd., St Louis, MO); Jack V Greiner, DO, PhD (Charles River Eye Associates, Winchester, MA); John A Hovanesian, MD (Harvard Eye Associates, Laguna Hills, CA); Mitchell A Jackson, MD (Jackson Eye, Lake Villa, IL); Stephen S Lane, MD (Associated Eye Care, Stillwater, MN); Parag A Majumudar, MD (Chicago Cornea Consultants, Ltd., Hoffman Estates, IL); William B Trattler, MD (Center for Excellence in Eye Care, Miami, FL).

\section{Disclosure}

$\mathrm{CAB}$ and $\mathrm{CAC}$ are employees of TearScience Inc. EJH serves on the Medical Advisory Board for TearScience.

\section{References}

1. Lemp MA, Baudouin C, Baum J, et al. The definition and classification of dry eye disease: report of the Definition and Classification Subcommittee of the International Dry Eye Workshop. Ocul Surf. 2007; 5(2):75-92.

2. Murube J. Andrew de Roetth (1893-1981): dacryologist who introduced the term dry eye. Ocul Surf. 2004;2(4):225-257.

3. Korb DR, Blackie CA. "Dry Eye" is the wrong diagnosis for millions. Optom Vis Sci. 2015;92(9):e350-e354.

4. Nichols KK. The international workshop on meibomian gland dysfunction: introduction. Invest Ophthalmol Vis Sci. 2011;52(4):1917-1921.

5. Nichols KK, Foulks GN, Bron AJ, et al. The international workshop on meibomian gland dysfunction: executive summary. Invest Ophthalmol Vis Sci. 2011;52(4):1922-1929.

6. Bron AJ, Tiffany JM, Gouveia SM, Yokoi N, Voon LW. Functional aspects of the tear film lipid layer. Exp Eye Res. 2004;78(3):347-360.

7. Mathers WD. Ocular evaporation in meibomian gland dysfunction and dry eye. Ophthalmology. 1993;100(3):347-351.

8. Mathers WD, Binarao G, Petroll M. Ocular water evaporation and the dry eye. A new measuring device. Cornea. 1993;12(4):335-340.

9. Mudgil P. Antimicrobial role of human meibomian lipids at the ocular surface. Invest Ophthalmol Vis Sci. 2014;55(11):7272-7277.

10. Rohit A, Willcox M, Stapleton F. Tear lipid layer and contact lens comfort: a review. Eye Contact Lens. 2013;39(3):247-253.

11. Ngo W, Caffery B, Srinivasan S, Jones LW. Effect of lid debridementscaling in Sjogren syndrome dry eye. Optom Vis Sci. 2015;92(9): e316-e320.

12. Schallhorn C, Schallhorn SC, Schallhorn JM. Effectiveness of an eyelid thermal pulsation procedure to treat recalcitrant dry eye symptoms after refractive surgery. Invest Ophthalmol Vis Sci. 2014;55:3694.

13. Wolff E. The mucocutaneous junction of the lidmargin and the distribution of the tear fluid. Trans Am Ophthalmol Soc. 1946;66:291-308.

14. Jackson M. Evaluation of thermal pulsation system treatment for meibomian gland dysfunction in cataract surgery patients. 2015 ASCRS ASOA Symposium and Congress; April 17-21, 2015; San Diego.

15. Napoli PE, Coronella F, Satta GM, Galantuomo MS, Fossarello M. Evaluation of the adhesive properties of the cornea by means of optical coherence tomography in patients with meibomian gland dysfunction and lacrimal tear deficiency. PLoS One. 2014;9(12):e115762.
16. Suhalim JL, Parfitt GJ, Xie Y, et al. Effect of desiccating stress on mouse meibomian gland function. Ocul Surf. 2014;12(1):59-68.

17. Blackie CA, Carlson AN, Korb DR. Treatment for meibomian gland dysfunction and dry eye symptoms with a single-dose vectored thermal pulsation: a review. Curr Opin Ophthalmol. 2015;26(4):306-313.

18. Gifford SR. Meibomian glands in chronic blepharoconjunctivitis. Am J Ophthalmol. 1921;4:489-494.

19. Korb DR, Henriquez AS. Meibomian gland dysfunction and contact lens intolerance. J Am Optom Assoc. 1980;51(3):243-251.

20. Lane SS, DuBiner HB, Epstein RJ, et al. A new system, the LipiFlow, for the treatment of meibomian gland dysfunction. Cornea. 2012; 31(4):396-404.

21. McCulley JP, Sciallis GF. Meibomian keratoconjunctivitis. Am J Ophthalmol. 1977;84(6):788-793.

22. Pflugfelder SC, Geerling G, Kinoshita S, et al. Management and therapy of dry eye disease: report of the Management and Therapy Subcommittee of the International Dry Eye Workshop. Ocul Surf. 2007;5:163-178.

23. Blackie CA, Solomon JD, Greiner JV, Holmes M, Korb DR. Inner eyelid surface temperature as a function of warm compress methodology. Optom Vis Sci. 2008;85(8):675-683.

24. Matsumoto Y, Dogru M, Goto E, et al. Efficacy of a new warm moist air device on tear functions of patients with simple meibomian gland dysfunction. Cornea. 2006;25:644-650.

25. Olson MC, Korb DR, Greiner JV. Increase in tear film lipid layer thickness following treatment with warm compresses in patients with meibomian gland dysfunction. Eye Contact Lens. 2003;29(2):96-99.

26. Korb DR, Blackie CA. Meibomian gland therapeutic expression: quantifying the applied pressure and the limitation of resulting pain. Eye Contact Lens. 2011;37:298-301.

27. Korb DR, Blackie CA. Restoration of meibomian gland functionality with novel thermodynamic treatment device - a case report. Cornea. 2010; 29(8):930-933.

28. Huang HW, Shih TC, Liauh CT. Predicting effects of blood flow rate and size of vessels in a vasculature on hyperthermia treatments using computer simulation. Biomed Eng Online. 2010;9:18.

29. Terada O, Chiba K, Senoo T, Obara Y. [Ocular surface temperature of meibomia gland dysfunction patients and the melting point of meibomian gland secretions]. Nippon Ganka Gakkai Zasshi. 2004;108(11): 690-693.

30. Finis D, Hayajneh J, König C, Borrelli M, Schrader S, Geerling G. Evaluation of an automated thermodynamic treatment (LipiFlow $®$ ) system for meibomian gland dysfunction: a prospective, randomized, observer-masked trial. Ocul Surf. 2014;12(2):146-154.

31. Finis D, Konig C, Hayajneh J, Borrelli M, Schrader S, Geerling G. Sixmonth effects of a thermodynamic treatment for MGD and implications of meibomian gland atrophy. Cornea. 2014;33:1265-1270.

32. Greiner JV. Long-term (12-month) improvement in meibomian gland function and reduced dry eye symptoms with a single thermal pulsation treatment. Clin Experiment Ophthalmol. 2013;41(6):524-530.

33. Greiner JV. Long-term (3 year) effects of a single thermal pulsation system treatment on meibomian gland function and dry eye symptoms. Eye Contact Lens. 2016;42(2):99-107.

34. Ngo W, Situ P, Keir N, Korb D, Blackie C, Simpson T. Psychometric properties and validation of the Standard Patient Evaluation of Eye Dryness questionnaire. Cornea. 2013;32:1204-1210.

35. Schiffman RM, Christianson MD, Jacobsen G, et al. Reliability and validity of the Ocular Surface Disease Index. Arch Ophthalmol. 2000; 118(5):615-621.

36. Geerling G, Tauber J, Baudouin C, et al. The international workshop on meibomian gland dysfunction: report of the subcommittee on management and treatment of meibomian gland dysfunction. Invest Ophthalmol Vis Sci. 2011;52(4):2050-2064.

37. Tomlinson A, Bron AJ, Korb DR, et al. The international workshop on meibomian gland dysfunction: report of the diagnosis subcommittee. Invest Ophthalmol Vis Sci. 2011;52(4):2006-2049.

38. Finis D, Pischel N, Schrader S, Geerling G. Evaluation of lipid layer thickness measurement of the tear film as a diagnostic tool for meibomian gland dysfunction. Cornea. 2013;32:1549-1553. 


\section{Publish your work in this journal}

Clinical Ophthalmology is an international, peer-reviewed journal covering all subspecialties within ophthalmology. Key topics include: Optometry; Visual science; Pharmacology and drug therapy in eye diseases; Basic Sciences; Primary and Secondary eye care; Patient Safety and Quality of Care Improvements. This journal is indexed on

Submit your manuscript here: http://www.dovepress.com/clinical-ophthalmology-journal
PubMed Central and CAS, and is the official journal of The Society of Clinical Ophthalmology (SCO). The manuscript management system is completely online and includes a very quick and fair peer-review system, which is all easy to use. Visit http://www.dovepress.com/ testimonials.php to read real quotes from published authors. 